\title{
Editorial: Special Issue on Stochastic Modelling of Reaction-Diffusion Processes in Biology
}

\author{
Radek Erban · Hans G. Othmer
}

Published online: 9 January 2014

(C) Society for Mathematical Biology 2013

Reaction-diffusion equations are used to model many biological processes, ranging from intracellular signaling, metabolic processes and gene control at the cellular level, to birth-death processes and random movement at the organism and population levels. There are two fundamental approaches to the mathematical modelling of these processes: deterministic (mean-field) models which lead to partial differential equations for concentrations of biochemical species or for densities of individuals, and stochastic models in which individual events of reaction or diffusion are followed. In some cases-such as linear processes or pure reaction processes based on mass action kinetics - one can prove that the latter description converges to the former in an appropriate 'large-number limit', but this is still an open question in general, as some of the papers herein illustrate.

Whenever the number of 'individuals' involved is small, stochastic effects can play an important role in the survival and spatiotemporal distribution of individuals. For instance, chemical reactions occur in discrete steps at the molecular level, the processes are inherently stochastic, and the inherent "irreproducibility" in these dynamics has been demonstrated experimentally for single-cell gene expression events (Ozbudak et al. 2002; Levsky and Singer 2003). Frequently stochastic effects simply add noise to an essentially deterministic output, but in others, such as asymmetric cell division, their role is essential.

\footnotetext{
R. Erban

Mathematical Institute, University of Oxford, Radcliffe Observatory Quarter, Woodstock Road, Oxford OX2 6GG, UK

e-mail: erban@maths.ox.ac.uk

H.G. Othmer $(\bowtie)$

School of Mathematics and Digital Technology Center, University of Minnesota, Minneapolis, MN 55455, USA

e-mail: othmer@math.umn.edu
} 
Stochastic reaction-diffusion simulations have been successfully used in a number of biological applications, including models of signal transduction in E. coli chemotaxis (Lipkow et al. 2005), MAPK pathway (Takahashi et al. 2010), oscillation of Min proteins in cell division (Fange and Elf 2006) and intracellular calcium dynamics (Flegg et al. 2013). Additional application areas are discussed in this Special Issue, including models of Hes1 gene regulatory network (Sturrock et al. 2013) and actin dynamics in filopodia (Erban et al. 2013). To study such a range of biological systems, a number of stochastic reaction-diffusion models have been introduced in the literature and several contributions in this Special Issue deal with advances in different mathematical and computational approaches.

One approach to computing solutions of stochastic reaction-diffusion models begins with a discretization of the spatial domain into compartments or voxels, just as in the numerical analysis of a continuum description (Erban et al. 2007; Kang et al. 2012; Wang et al. 2013). This approach can be mathematically described by the reaction-diffusion master equation in which diffusion between computational cells is modelled as a stochastic jump process. In this Special Issue, Sturrock et al. (2013) use this methodology to study the Hes1 gene regulatory network. They follow the URDME implementation (Drawert et al. 2012) of compartment-based models to investigate oscillatory dynamics. The advantage of URDME is that it uses unstructured compartments (meshes) and therefore can more accurately describe detailed geometries of biological systems (Engblom et al. 2009).

Another important generalization of compartment-based models is to the modelling of one-dimensional structures such as microtubes and actin filaments that are embedded in a three-dimensional discretization of intracellular space. This method is developed herein by Wang et al. (2013), who apply it to modelling binding of transcription factors to DNA. Other implementations of compartment-based approaches include the software package MesoRD (Hattne et al. 2005) and the finite state projection algorithm for the stochastic reaction-diffusion master equation (Drawert et al. 2010).

The selection of compartment size in a discretized spatial domain is another important area of current research. Estimates for the minimal compartment size can be derived using a range of different methods (Erban and Chapman 2009; Isaacson 2009; Hellander et al. 2012b). In this Special Issue, the compartment size selection is discussed by $\mathrm{Hu}$ et al. (2013), who also present a stochastic simulation algorithm for compartmentalized systems in which reactions are aggregated into equivalence classes and computational cells are searched via an optimized tree structure. Another technique for analysis of compartment-based models, called linear noise analysis, is reviewed by McKane et al. (2013), who show how the stochastic amplification of a Turing instability (Murray 2002) gives rise to spatial and temporal patterns which may be understood within the linear noise approximation.

A more detailed approach to stochastic reaction-diffusion modelling is based on molecular-based Brownian dynamics simulations, which compute trajectories of individual molecules. This methodology has been implemented in the simulation software packages Smoldyn (Andrews and Bray 2004), MCell (Stiles and Bartol 2001) and Green's Function Reaction Dynamics (GFRD) (van Zon and ten Wolde 2005; Takahashi et al. 2010). Simulating systems which only include zero-order and firstorder reactions are relatively straightforward in all stochastic reaction-diffusion 
methods. In fact, one can show that the mean-field model predicts the average behavior of such systems (Gadgil et al. 2005; Kang et al. 2012). More interesting system dynamics can be observed whenever some chemical species undergo bimolecular reactions. A fundamental assumption here is that a reaction occurs whenever the participating molecules are within a certain distance, thus localizing bimolecular reactions (Erban and Chapman 2009). In this Special Issue, Agbanusi and Isaacson (2013) study the relationship between two common models of bimolecular reactions, and show that they are equivalent in a certain limit. They also apply their theory to a protein diffusing to a fixed DNA binding site. Bimolecular reactions also have to be handled with care whenever they are reversible (Lipkova et al. 2011; Khokhlova and Agmon 2012) or the reaction occurs at the domain boundary (Erban and Chapman 2007).

Molecular-based models can also capture steric effects due to, e.g., macromolecular crowding or confined geometries. Bruna and Chapman (2013) study the applications of these methods to mathematical modelling of ion channels in their contribution. There have been also recent developments in combining different stochastic reaction-diffusion approaches together in a multiscale framework (Ferm et al. 2010; Flegg et al. 2012; Hellander et al. 2012a) or combining stochastic models with meanfield descriptions (Flekkøy et al. 2001; Ferm et al. 2010; Franz et al. 2013). In this Special Issue, Erban et al. (2013) investigate two multiscale (hybrid) stochastic reaction-diffusion models of actin dynamics in a filopodium. They compare these techniques with a fully molecular-based model which they also develop in their paper.

The articles in this Special Issue were contributed by participants in the workshop on "Stochastic Modelling of Reaction-Diffusion Processes" which took place in July 2012 in Oxford. ${ }^{1}$ This workshop attracted over 70 participants from around the world and brought together three (overlapping) groups of researchers: (i) researchers who use stochastic reaction-diffusion models to answer specific biological questions; (ii) mathematicians who analyse stochastic reaction-diffusion algorithms; and (iii) developers of stochastic reaction-diffusion software. This Special Issue provides a snapshot of current research in this field to a wider mathematical biology audience.

\section{References}

Agbanusi, I., \& Isaacson, S. (2013). A comparison of bimolecular reaction models for stochastic reactiondiffusion systems. Bull. Math. Biol. doi:10.1007/s11538-013-9833-6.

Andrews, S., \& Bray, D. (2004). Stochastic simulation of chemical reactions with spatial resolution and single molecule detail. Phys. Biol., 1, 137-151.

Bruna, M., \& Chapman, S. J. (2013). Diffusion of finite-size particles in confined geometries. Bull. Math. Biol. doi:10.1007/s11538-013-9847-0.

\footnotetext{
${ }^{1}$ Funding for this workshop was provided by the NSF-funded Mathematical Biosciences Institute, Ohio State University, US; the Oxford Centre for Collaborative Applied Mathematics (OCCAM) and the Mathematical Institute, University of Oxford, United Kingdom; and the Leverhulme Trust. The workshop website is available at http://people.maths.ox.ac.uk/erban/workshop/.
} 
Drawert, B., Lawson, M., Petzold, L., \& Khammash, M. (2010). The diffusive finite state projection algorithm for efficient simulation of the stochastic reaction-diffusion master equation. J. Chem. Phys., 132, 074101 .

Drawert, B., Engblom, S., \& Hellander, A. (2012). URDME: a modular framework for stochastic simulation of reaction-transport processes in complex geometries. BMC Syst. Biol., 6, 76.

Engblom, S., Ferm, L., Hellander, A., \& Lötstedt, P. (2009). Simulation of stochastic reaction-diffusion processes on unstructured meshes. SIAM J. Sci. Comput., 31, 1774-1797.

Erban, R., \& Chapman, S. J. (2007). Reactive boundary conditions for stochastic simulations of reactiondiffusion processes. Phys. Biol., 4(1), 16-28.

Erban, R., \& Chapman, S. J. (2009). Stochastic modelling of reaction-diffusion processes: algorithms for bimolecular reactions. Phys. Biol., 6(4), 046001.

Erban, R., Chapman, S. J., \& Maini, P. (2007). A practical guide to stochastic simulations of reactiondiffusion processes. 35 pages. http://arxiv.org/abs/0704.1908.

Erban, R., Flegg, M., \& Papoian, G. (2013). Multiscale stochastic reaction-diffusion modelling: application to actin dynamics in filopodia. Bull. Math. Biol. doi:10.1007/s11538-013-9844-3.

Fange, D., \& Elf, J. (2006). Noise-induced Min phenotypes in E. coli. PLoS Comput. Biol., 2(6), 637-648.

Ferm, L., Hellander, A., \& Lötstedt, P. (2010). An adaptive algorithm for simulation of stochastic reactiondiffusion processes. J. Comput. Phys., 229, 343-360.

Flegg, M., Chapman, J., \& Erban, R. (2012). The two-regime method for optimizing stochastic reactiondiffusion simulations. J. R. Soc. Interface, 9(70), 859-868.

Flegg, M., Rüdiger, S., \& Erban, R. (2013). Diffusive spatiotemporal noise in a first-passage time model for intracellular calcium release. J. Chem. Phys., 138, 154103.

Flekkøy, E., Feder, J., \& Wagner, G. (2001). Coupling particles and fields in a diffusive hybrid model. Phys. Rev. E, 64, 066302.

Franz, B., Flegg, M., Chapman, J., \& Erban, R. (2013). Multiscale reaction-diffusion algorithms: PDEassisted Brownian dynamics. SIAM J. Appl. Math., 73(3), 1224-1247.

Gadgil, C., Lee, C., \& Othmer, H. (2005). A stochastic analysis of first-order reaction networks. Bull. Math. Biol., 67, 901-946.

Hattne, J., Fange, D., \& Elf, J. (2005). Stochastic reaction-diffusion simulation with MesoRD. Bioinformatics, 21(12), 2923-2924.

Hellander, A., Hellander, S., \& Lötstedt, P. (2012a). Coupled mesoscopic and microscopic simulation of stochastic reaction-diffusion processes in mixed dimensions. Multiscale Model. Simul., 10(2), 585611.

Hellander, S., Hellander, A., \& Petzold, L. (2012b). Reaction-diffusion master equation in the microscopic limit. Phys. Rev. E, 85, 042901.

Hu, J., Kang, H., \& Othmer, H. (2013). Stochastic analysis of reaction-diffusion processes. Bull. Math. Biol. doi:10.1007/s11538-013-9849-y.

Isaacson, S. (2009). The reaction-diffusion master equation as an asymptotic approximation of diffusion to a small target. SIAM J. Appl. Math., 70(1), 77-111.

Kang, H. W., Zheng, L., \& Othmer, H. G. (2012). A new method for choosing the computational cell in stochastic reaction-diffusion systems. J. Math. Biol., 65(6-7), 1017-1099.

Khokhlova, S., \& Agmon, N. (2012). Green's function for reversible geminate reaction with volume reactivity. J. Chem. Phys., 137(5), 184103.

Levsky, J., \& Singer, R. (2003). Gene expression and the myth of the average cell. Trends Cell Biol., 13(1), 4-6.

Lipkova, J., Zygalakis, K., Chapman, J., \& Erban, R. (2011). Analysis of Brownian dynamics simulations of reversible bimolecular reactions. SIAM J. Appl. Math., 71(3), 714-730.

Lipkow, K., Andrews, S., \& Bray, D. (2005). Simulated diffusion of phosphorylated CheY through the cytoplasm of Escherichia coli. J. Bacteriol., 187(1), 45-53.

McKane, A., Biancalani, T., \& Rogers, T. (2013). Stochastic pattern formation and spontaneous polarization: the linear noise approximation and beyond. Bull. Math. Biol. doi:10.1007/s11538-013-9827-4.

Murray, J. (2002). Mathematical Biology. Berlin: Springer.

Ozbudak, E., Thattai, M., Kurtser, I., Grossman, A., \& van Oudenaarden, A. (2002). Regulation of noise in the expression of a single gene. Nat. Genet., 31, 69-73.

Stiles, J., \& Bartol, T. (2001). Monte Carlo methods for simulating realistic synaptic microphysiology using MCell. In E. Schutter (Ed.), Computational neuroscience: realistic modeling for experimentalists (pp. 87-127). Boca Raton: CRC Press. 
Sturrock, M., Hellander, A., Aldakheel, S., Petzold, L., \& Chaplain, M. A. J. (2013). The role of dimerisation and nuclear transport in the Hes1 gene regulatory network. Bull. Math. Biol. doi:10.1007/ s11538-013-9842-5.

Takahashi, K., Tanase-Nicola, S., \& ten Wolde, P. (2010). Spatiotemporal correlations can drastically change the response of a MAPK pathway. Proc. Natl. Acad. Sci. USA, 107, 19820-19825.

van Zon, J., \& ten Wolde, P. (2005). Green's-function reaction dynamics: a particle-based approach for simulating biochemical networks in time and space. J. Chem. Phys., 123, 234910.

Wang, S., Elf, J., Hellander, S., \& Lötstedt, P. (2013). Stochastic reaction-diffusion processes with embedded lower dimensional structures. Bull. Math. Biol. doi:10.1007/s11538-013-9910-x. 